Pacific Journal of Mathematics

ENUMERATION OF SELF-DUAL CONFIGURATIONS 


\title{
ENUMERATION OF SELF-DUAL CONFIGURATIONS
}

\author{
E. M. PAlmer AND R. W. Robinson
}

\begin{abstract}
A variety of combinatorial structures are self-dual in the sense that opposite elements have opposite properties. We provide a general enumeration theorem for these which has a number of interesting applications including the enumeration of self-dual boolean functions and 2-colorings of the vertices of polyhedra in which opposite vertices have different colors. Our method involves a modification of Pólya's enumeration theorem.
\end{abstract}

Introduction. Suppose that each of the twenty vertices of a dodecahedron is given one of two colors, say black or white. Then there are the following three enumeration problems that arise for these 2-colored configurations:

(1) What is the number of 2-colorings with an equal number of points of each color?

(2) What is the number of self-complementary 2-colorings, in which the configuration is unchanged on interchanging the colors?

(3) What is the number of self-dual 2-colorings, in which opposite points have different colors?

Note that we always regard two such colorings as equivalent if one can be brought into coincidence with the other by a rotation or reflection of the dodecahedron.

The answer to the first question, namely 1648, can be obtained by applying Pólya's enumeration theorem [12] to the cycle index of the automorphism group of the dodecahedron. The number of self-complementary colorings is 140 and this can be calculated using the approach of Read [13] in determining the number of self-complementary graphs. Finally, the number of self-dual colorings is 20 , and this latter invariant can be computed using the modification of Pólya's theorem presented in this paper. Our approach is reminiscent of the enumeration of orientations of a graph [5, p. 128] where the appropriate permutation group is expressed in terms of two sets of variables. The resulting enumeration theorem can be used in a variety of interesting situations. We present applications to necklaces, polyhedra, and boolean functions.

\section{Enumeration Theorem for Self-Dual Configurations}

In this section we introduce our notation, provide a brief review of Pólya's enumeration theorem [12] and conclude with our modification for 
self-dual configurations. The presentation is virtually self-contained but a more substantial background for the theory including proofs can be found in the book Graphical Enumeration [5].

We consider the functions from $X$ to $Y$ and a permutation group $A$ with object set $X$. Then two functions $f$ and $g$ are $A$-equivalent if there is a permutation $\alpha$ and $A$ such that $f(\alpha x)=g(x)$ for all $x$ in $X$. For the sake of simplicity we initially consider the important special case that $Y$ is a 2 element set, e.g. $Y=\{0,1\}$. Statements of the results for general $Y$ are given at the end of the section.

An example is provided by taking as $X$ the set of 6 vertices of the octahedron, while $A$ is the octahedral group of order 48 and $Y$ is the set $\{0,1\}$ of two colors, zero for white and 1 for black. Then each function represents a coloring of the vertices and the $A$-equivalence classes correspond to the colorings in which the vertices are no longer distinguished by their labels.

Pólya's theorem can be used to enumerate these colorings with the number of white points as an enumeration parameter. The enumeration makes use of the cycle index, denoted by $Z(A)$, of the permutation group $A$. For each permutation $\alpha$ in $A$, and each positive integer $k$, let $j_{k}(\alpha)$ be the number of cycles of length $k$ in the disjoint cycle decomposition of $\alpha$. Then $Z(A)$ is the polynomial in the variables $a_{1}, a_{2}, a_{3}, \ldots$ defined by

$$
Z(A)=\frac{1}{|A|} \sum_{\alpha \in A} \prod_{k} a_{k}^{j_{k}(\alpha)}
$$

For example, the cycle index of the octahedral group acting on the 6 vertices of the octahedron is

$$
\begin{aligned}
\frac{1}{48}\left\{\left(a_{1}^{6}+6 a_{1}^{2} a_{4}\right.\right. & \left.+3 a_{1}^{2} a_{2}^{2}+8 a_{3}^{2}+6 a_{2}^{3}\right) \\
& \left.+\left(a_{2}^{3}+6 a_{2} a_{4}+3 a_{1}^{4} a_{2}+8 a_{6}+6 a_{1}^{2} a_{2}^{2}\right)\right\} .
\end{aligned}
$$

TheORem 1. (Pólya) The number of A-equivalence classes of functions $f: X \rightarrow\{0,1\}$, where $\left|f^{-1}(1)\right|=r$, is the coefficient of $z^{r}$ in the polynomial obtained from $Z(A)$ by substituting $1+z^{k}$ for each variable $a_{k}$; symbolically this polynomial is $Z(A, 1+z)$.

On applying this result to our example we obtain

$$
1+z+2 z^{2}+2 z^{3}+2 z^{4}+z^{5}+z^{6}
$$


as the enumerator of 2-colorings of the vertices of the octahedron. The coefficient of $z^{r}$ is the number of 2-colorings with $r$ black vertices and $6-r$ white vertices. The middle coefficient is the number of colorings with an equal number of vertices of each color. The two colorings with 3 points of each color are displayed in Figure 1.
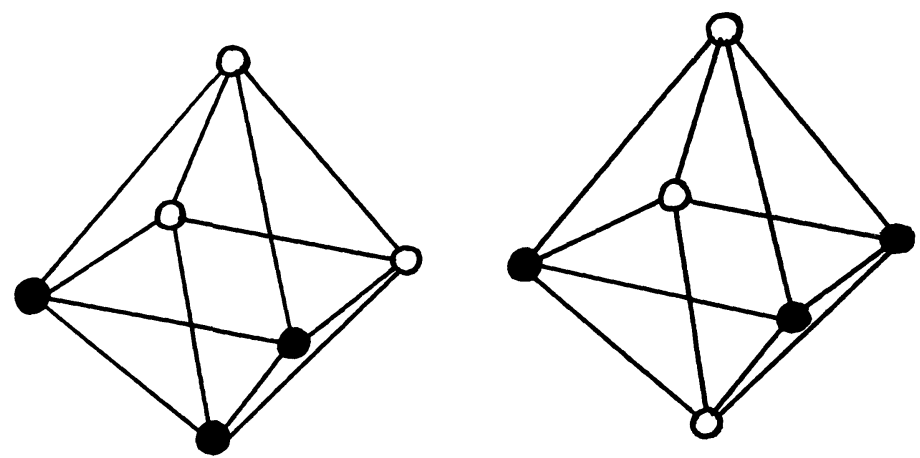

FIGURE 1. The colorings of the octahedron with 3 points of each color.

Now suppose that involutions on $X$ and $Y$ are given. Each is denoted by an asterisk and we assume that the following commutative law is satisfied by all $\alpha$ in $A$ and $x$ in $X$ :

$$
\alpha\left(x^{*}\right)=(\alpha x)^{*} .
$$

In our example, where $X$ is the set of vertices of the octahedron, we shall consider the involution which interchanges opposite vertices of $X$ and the one that interchanges the two colors in $Y$.

The complement $\bar{f}$ of the function $f$ is defined by

$$
\bar{f}(x)=f(x)^{*}
$$

for all $x$. A function is self-complementary if it is $A$-equivalent to its complement. Each of the two colorings of the octahedron in Figure 1 is seen to be self-complementary.

Next we state the theorem for enumerating self-complementary functions with range $Y=\{0,1\}$ and the transposition on $Y$ as its involution.

THEOREM 2. The number of A-equivalence classes of self-complementary functions is obtained by setting the variables $a_{k}$ in the cycle index of $A$ equal to 0 if $k$ is odd and 2 if $k$ is even; symbolically this number is $Z(A ; 0,2,0,2, \ldots)$.

This result follows from de Bruijn's generalization of Pólya's theorem [2, p. 179] and was used by Read to count self-complementary graphs and digraphs [13]. It appears that the result was known to Redfield, as 
evidenced by an example in his paper [14, p. 443]. The basic idea has been extended by de Bruijn to an arbitrary number of colors which are interchangeable under some fixed permutation [3], thus permitting the enumeration of those functions which are left in the same equivalence class by this permutation.

The dual $f^{*}$ of the function $f$ is defined by

$$
f^{*}(x)=f\left(x^{*}\right)
$$

for all $x$. In our example, the dual of a coloring of the octahedron is obtained by assigning to each vertex the color of the opposite vertex.

A function $f$ is self-dual if

$$
f\left(x^{*}\right)=f(x)^{*}
$$

for all $x$. Thus, in the example, a coloring is self-dual if opposite points have opposite colors. Hence the first octahedron in Figure 1 is self-dual while the second is not. Note that, as in this example, whenever the involution on $X$ is also an element of $A$, self-dual functions are also self-complementary.

The problem of enumerating $A$-equivalence classes of self-dual functions can be handled by introducing a modification of the cycle index of $A$ which keeps track of the cycles in the permutations which mix elements $x$ of $X$ with $x^{*}$. Consider any permutation $\alpha$ in $A$ and let $c=\left(x_{1} x_{2} \cdots x_{k}\right)$ be one of the cycles in its disjoint cycle decomposition. It follows from the commutativity of ${ }^{*}$ with $\alpha$ that $c^{*}=\left(x_{1}^{*} x_{2}^{*} \cdots x_{k}^{*}\right)$ is another such cycle called the dual of $c$. If $c=c^{*}$, it is self-dual. We denote by $Z^{*}\left(A ; s_{k}, t_{k}^{2}\right)$ that modification of $Z(A)$ in which the variables $s_{k}$ correspond to the self-dual cycles of $k$ elements, while $t_{k}^{2}$ corresponds to a pair of different dual cycles.

THEOREM 3. The number of A-equivalence classes of self-dual functions $f: X \rightarrow\{0,1\}$, with transposition as the involution on $\{0,1\}$, is obtained from $Z^{*}\left(A ; s_{k}, t_{k}^{2}\right)$ by setting $s_{k}=0$ and $t_{k}^{2}=2$ for all $k$; symbolically this number is $Z^{*}(A ; 0,2)$.

In our example we find this modified cycle index to be

$$
\begin{aligned}
\frac{1}{48}\left\{\left(t_{1}^{6}+6 t_{1}^{2} s_{4}+\right.\right. & \left.3 t_{1}^{2} s_{2}^{2}+8 t_{3}^{2}+6 t_{2}^{2} s_{2}\right) \\
& \left.+\left(s_{2}^{3}+6 s_{2} s_{4}+3 t_{1}^{4} s_{2}+8 s_{6}+6 t_{1}^{2} t_{2}^{2}\right)\right\} .
\end{aligned}
$$

On setting $s_{k}=0$ and $t_{k}^{2}=2$ for all $k$ we obtain the number 1 . The single self-dual coloring of the octahedron is the first graph in Figure 1. 
The proof consists of a straightforward application of Burnside's Lemma [5, p. 38]. The group $A$ is represented as acting on the self-dual functions from $X$ to $Y$. For each $\alpha$ in $A$ its representation $\alpha^{\prime}$ is defined by

$$
\left(\alpha^{\prime} f\right)(x)=f(\alpha x)
$$

for all $x$ in $X$ and all self-dual functions $f$. It is easily checked that $\alpha^{\prime} f$ is also self-dual. The $A$-equivalence classes of self-dual functions are just the orbits of self-dual functions determined by this representation of $A$. On applying Burnside's Lemma we have the result that the number of these orbits is

$$
\frac{1}{|A|} \sum_{\alpha \in A} N(\alpha)
$$

where $N(\alpha)$ is the number of self-dual functions left fixed by $\alpha^{\prime}$. We now express $N(\alpha)$ in terms of the cycle decomposition of $\alpha$.

Suppose $f$ is self-dual and is fixed by $\alpha^{\prime}$, i.e. $\alpha^{\prime} f=f$. Then $f(\alpha x)=f(x)$ for all $x$ and so $f$ must be constant on the elements of each cycle of $\alpha$. In particular, if $x$ is an element of a self-dual cycle of $\alpha$, so is $x^{*}$ and hence $f(x)=f\left(x^{*}\right)$. On the other hand, since $f$ is self-dual, we have $f\left(x^{*}\right)=f(x)^{*}$ by definition. It follows that $f(x)=f(x)^{*}$, contrary to the fact that the involution ${ }^{*}$ on $Y$ has no fixed points. Therefore $N(\alpha)=0$ whenever $\alpha$ has any self-dual cycles.

Now consider a dual pair of cycles $c$ and $c^{*}$ of $\alpha$. There are exactly two ways in which a self-dual function $f$ can be constant on these cycles: either $f$ sends the elements of $c$ to 0 and those of $c^{*}$ to 1 or vice versa.

For each $k=1,2,3, \ldots$, let $u_{k}$ be the number of self-dual cycles in $\alpha$ of length $k$ while $v_{k}$ is the number of dual pairs of cycles of length $k$. The observations made above prove that

$$
N(\alpha)=\prod_{k} 0^{u_{k}} 2^{v_{k}}
$$

Here we use the convention that $a^{0}=1$ even when $a=0$. On summing this expression for $N(\alpha)$ over all $\alpha$ and dividing by $|A|$ we have the number of orbits of self-dual functions expressed symbolically as $Z^{*}(A ; 0,2)$.

In a more general context we need not require $Y=\{0,1\}$. Suppose instead that the * operation on $Y$ consists of $m$ transpositions and $n$ fixed points, so that the preceding theorems correspond to the case $m=1$, $n=0$. Then Theorems 1, 2, and 3 are generalized as follows. 
THEOREM 1'. The number of A-equivalence classes of functions $f: X \rightarrow Y$ with equal numbers of colors from each dual pair in $Y$ is the constant term in

$$
Z\left(A ; n+\sum_{i=1}^{m}\left(x_{i}+x_{i}^{-1}\right)\right) .
$$

THEOREM 2'. The number of A-equivalence classes of self-complementary functions is

$$
Z(A ; n, n+2 m, n, n+2 m, \ldots) .
$$

THEOREM 3'. The number of A-equivalence classes of self-dual functions is

$$
Z^{*}(A ; n, n+2 m) \text {. }
$$

Theorems $1^{\prime}, 2^{\prime}$ and $3^{\prime}$ can be proved in much the same way as Theorems 1, 2, and 3.

\section{Applications}

The enumeration theorems for self-dual configurations can be used to count a variety of interesting structures. We shall discuss the particular cases of necklaces, polyhedra, and boolean functions.

1. Necklaces. We count 2-colored necklaces with $n=2 m$ beads in which opposite beads have different colors. When $n=10$, there are four such self-dual necklaces and they are displayed in the next figure:
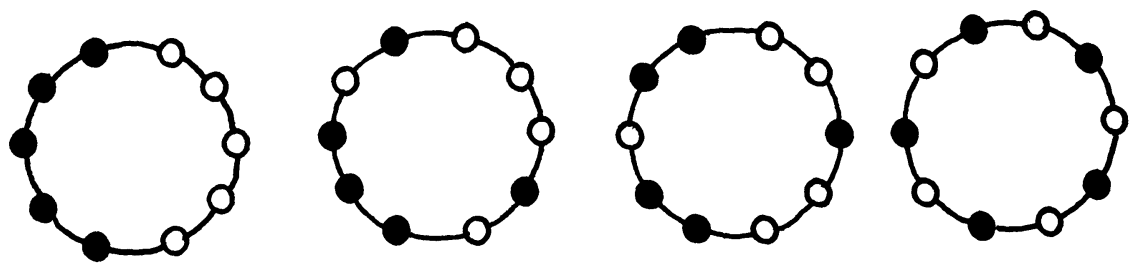

FIGURE 2. The four self-dual 2-colored necklaces of 10 beads

The duality involution on the beads of a necklace is given by a $180^{\circ}$ rotation. Of course the involution on the colors is the transposition.

Necklaces of $n=2 m$ beads can be enumerated by applying Pólya's theorem to the dihedral group $D_{2 m}$ on $n$ objects. Similarly Theorem 3 can be applied once $Z^{*}\left(D_{2 m}\right)$ is known. For the cyclic group $C_{2 m}$, the modified cycle index is

$$
Z^{*}\left(C_{2 m} ; s_{k}, t_{k}^{2}\right)=\frac{1}{2 m} \sum_{2 \nmid k \mid m} \varphi(k) t_{k}^{2 m / k}+\frac{1}{2 m} \sum_{2 k \mid m} \varphi(2 k) s_{2 k}^{m / k},
$$


where $\varphi$ denotes the Euler totient function. This formula follows quickly from the observations that cycles in $C_{2 m}$ are self-dual if and only if they have even length.

For the dihedral group we have

$$
\begin{aligned}
Z^{*}\left(D_{2 m} ; s_{k}, t_{k}^{2}\right)= & \frac{1}{2} Z^{*}\left(C_{2 m} ; s_{k}, t_{k}^{2}\right) \\
& +\frac{1}{4} \begin{cases}t_{2}^{m}+t_{1}^{2} s_{2} t_{2}^{m-2} & \text { if } m \text { is even, } \\
s_{2} t_{2}^{m-1}+t_{1}^{2} t_{2}^{m-1} & \text { if } m \text { is odd }\end{cases}
\end{aligned}
$$

It follows from Theorem 3 that on setting $t_{k}^{2}=2$ and $s_{k}=0$ for all $k$ in the latter expression, we obtain the number of self-dual, 2-colored necklaces on $n=2 m$ beads. On carrying out the substitution, this number can be written

$$
\frac{1}{4 m} \sum_{2 \nmid k \mid m} \varphi(k) 2^{m / k}+2^{\lfloor(m-3) / 2\rfloor}
$$

where $\lfloor x\rfloor$ is the greatest integer in $x$. The numerical values of this sum for $2 \leq n \leq 20$ are presented in Table 1. Also included in the table for purposes of comparison are the corresponding values for necklaces with equal numbers of black and white beads, and for necklaces which are self-complementary under the interchange of black and white. The former is found by Theorem 1 to be the coefficient of $x^{m}$ in

$$
Z\left(D_{2 m} ; 1+x, 1+x^{2}, \ldots\right)
$$

which can be put more explicitly as

$$
\frac{1}{4 m} \sum_{k \mid m} \varphi(k)\left(\begin{array}{c}
2 m / k \\
m / k
\end{array}\right)+\frac{1}{2}\left(\begin{array}{c}
2\lfloor m / 2\rfloor \\
\lfloor m / 2\rfloor
\end{array}\right) .
$$

By Theorem 2 one finds that the number of self-complementary 2-colored necklaces is

$$
Z\left(D_{2 m} ; 0,2,0,2, \ldots\right)
$$

which simplifies to

$$
\frac{1}{4 m} \sum_{k \mid m} \varphi(2 k) 2^{m / k}+2^{m-2} .
$$




\begin{tabular}{r|c|c|c}
$2 m$ & self-dual & self-complementary & $m$ beads of each color \\
\hline 2 & 1 & 1 & 1 \\
4 & 1 & 2 & 2 \\
6 & 2 & 3 & 3 \\
8 & 2 & 6 & 8 \\
10 & 4 & 10 & 16 \\
12 & 5 & 20 & 50 \\
14 & 9 & 37 & 133 \\
16 & 12 & 74 & 440 \\
18 & 23 & 143 & 1387 \\
20 & 34 & 284 & 4752
\end{tabular}

TABLE 1. 2-colored necklaces with $2 m$ beads.

The numbers of self-dual colorings of necklaces are closely related to two other sequences. A tournament is locally transitive if for each point $v$ the subtournament induced by the points adjacent from $v$ is transitive as well as the subtournament induced by the points adjacent to $v$. Brouwer [1] has enumerated these by showing that they correspond to certain shift registers [4, p. 172]. In our notation, the numbers of locally transitive tournaments of order $m$ is $Z^{*}\left(C_{2 m} ; 0,2\right)$, i.e. self-dual 2-colorings of necklaces in which reflection is not permitted.

2. Polyhedra. Next we consider the problem of coloring with two colors the lattice points which are solutions of the equation $x^{2}+y^{2}+z^{2}$ $\leq m$ so that opposite points have different colors. It is easily seen that this problem as well as many others similar to it, can be handled by Theorem 3. The main difficulty then is to calculate the cycle indexes of the groups involved and to express them in the two variables $s_{k}$ and $t_{k}$. The various configurations enumerated correspond in solid state chemistry to arrangements of point charges having zero electric field gradient and are used in the study of quadrupole splitting in the Mössbauer spectra of certain ionic crystals [8].

First we shall classify the integer solutions of $x^{2}+y^{2}+z^{2}=m$. If $(a, b, c)$ is a solution, then the opposite or dual vertex $(-a,-b,-c)$ is also a solution. When $m=1$, there are exactly 6 solutions, namely $(1,0,0)$, $(0,1,0),(0,0,1),(-1,0,0),(0,-1,0)$, and $(0,0,-1)$, and these solutions constitute the vertices of the octahedron. Such solutions which have two coordinates equal to zero, we call type I solutions. There are five others and each has associated with it a different polyhedron. These are listed in Table 2. The rotations and reflections of the sphere which permute the 


\begin{tabular}{rlcc} 
Type & \multicolumn{1}{c}{$\begin{array}{c}\text { Polyhedron } \\
\text { Number of } \\
\text { Vertices }\end{array}$} & $\begin{array}{c}\text { Typical } \\
\text { Vertex }\end{array}$ \\
I & octahedron & 6 & $(a, 0,0)$ \\
II & cuboctahedron & 12 & $(a, a, 0)$ \\
III & cube & 8 & $(a, a, a)$ \\
IV & truncated & 24 & $(a, b, 0)$ \\
& $\begin{array}{l}\text { octahedron } \\
\text { truncated }\end{array}$ & 24 & $(a, a, b)$ \\
V & $\begin{array}{l}\text { cube } \\
\text { truncated } \\
\text { VI }\end{array}$ & 48 & $(a, b, c)$
\end{tabular}

TABLE 2. The six types of polyhedra whose vertices are solutions of $x^{2}+y^{2}+z^{2}=m$.

solutions of one type form a transitive representation of the octahedral group of order 48. It is easy to calculate directly all the terms in the cycle indexes of these representations and we have collected this information in Table 3. Recall that the variables $s_{k}$ correspond to self-dual cycles in which $k / 2$ opposite pairs of vertices are permuted, while $t_{k}^{2}$ refers to a pair of cycles, each permuting the $k$ opposite vertices of the other. Note that type I solutions, which form the vertices of a regular octahedron, give rise to the cycle index which was taken as an example for the enumeration theorem for self-dual configurations. The type III solutions form a cube and the corresponding cycle index terms in Table 3 agree with the cycle index formula worked out for boolean functions of three arguments in the next section.

\begin{tabular}{|c|c|c|c|c|c|c|c|c|c|c|}
\hline \multirow{2}{*}{$\begin{array}{c}\text { Number of } \\
\text { permutations }\end{array}$} & \multicolumn{5}{|c|}{ rotations } & \multicolumn{5}{|c|}{ reflections } \\
\hline & 1 & 6 & 3 & 8 & 6 & 1 & 6 & 3 & 8 & 6 \\
\hline I & $t_{1}^{6}$ & $s_{4} t_{1}^{2}$ & $s_{2}^{2} t_{1}^{2}$ & $t_{3}^{2}$ & $s_{2} t_{2}^{2}$ & $s_{2}^{3}$ & $s_{2} s_{4}$ & $s_{2} t_{1}^{4}$ & $s_{6}$ & $t_{1}^{2} t_{2}^{2}$ \\
\hline II & $t_{1}^{12}$ & $s_{4} t_{4}^{2}$ & $s_{2}^{2} t_{2}^{4}$ & $t_{3}^{4}$ & $s_{2} t_{1}^{2} t_{2}^{4}$ & $s_{2}^{6}$ & $s_{4} t_{4}^{2}$ & $t_{1}^{4} t_{2}^{4}$ & $s_{6}^{2}$ & $s_{2} t_{1}^{2} t_{2}^{4}$ \\
\hline III & $t_{1}^{8}$ & $t_{4}^{2}$ & $t_{2}^{4}$ & $t_{1}^{2} t_{3}^{2}$ & $s_{2}^{2} t_{2}^{2}$ & $s_{2}^{4}$ & $t_{4}^{2}$ & $t_{2}^{4}$ & $s_{2} s_{6}$ & $t_{1}^{4} t_{2}^{2}$ \\
\hline IV & $t_{1}^{24}$ & $s_{4}^{2} t_{4}^{4}$ & $s_{2}^{4} t_{2}^{8}$ & $t_{3}^{8}$ & $t_{2}^{12}$ & $s_{2}^{12}$ & $s_{4}^{2} t_{4}^{4}$ & $t_{1}^{8} t_{2}^{8}$ & $s_{6}^{4}$ & $t_{2}^{12}$ \\
\hline V & $t_{1}^{24}$ & $t_{4}^{6}$ & $t_{2}^{12}$ & $t_{3}^{8}$ & $s_{2}^{2} t_{2}^{10}$ & $s_{2}^{12}$ & $t_{4}^{6}$ & $t_{2}^{12}$ & $s_{6}^{4}$ & $t_{1}^{4} t_{2}^{10}$ \\
\hline VI & $t_{1}^{48}$ & $t_{4}^{12}$ & $t_{2}^{24}$ & $t_{3}^{16}$ & $t_{2}^{24}$ & $s_{2}^{24}$ & $t_{4}^{12}$ & $t_{2}^{24}$ & $s_{6}^{8}$ & $t_{2}^{24}$ \\
\hline
\end{tabular}

TABLE 3. Cycle structures of six types of representation of the octahedral group.

Here are some of the details which explain the entries in this table for the cube.

Column 1 . The identity rotation. All 8 vertices are fixed so the entry is $t_{1}^{8}$. 
Column 2. There are 2 rotations of order 4 about each of the 3 axes through opposite faces. Hence there are 6 permutations. Each has 2 cycles of order 4.

Column 3. The 3 squares of the rotations of order 4 . Each has 4 cycles of order 2.

Column 4. There are two rotations of order 3 about each of 4 axes through opposite pairs of points. The two opposite points on this axis are fixed and the others move in cycles of order 3 . Hence these 8 permutations are each of type $t_{1}^{2} t_{3}^{2}$.

Column 5. There is one rotation of order 2 about each of 6 pairs of opposite edges. The vertices are all moved in cycles of order 2 but two of these are self-dual. Hence there are 6 permutations described by $s_{2}^{2} t_{2}^{2}$.

The next 5 columns for reflections are determined by the rotations followed by the reflection which interchanges opposite vertices. Then the number of permutations is the same as for the corresponding number of rotations. A few simple observations facilitate the compilation of the entries for the reflections. They can all be obtained from the corresponding rotation entry by the following rules:

(a) $t_{2 k+1}^{2}$ always becomes $s_{2(2 k+1)}$ and vice versa.

(b) $t_{2 k}^{2}$ and $s_{4 k}$ are always unchanged.

To show how the enumeration theorem for self-dual configurations can be applied to count self-dual colorings, consider the solutions of $x^{2}+y^{2}+z^{2} \leq 4$. There are two solutions of type I, and one each of types II and III. Using Table 3, we can express the contributing portion of the cycle index of the octahedral group represented on all the vertices of these solutions as follows:

$$
\frac{1}{48}\left\{\left(t_{1}^{6}\right)^{2} t_{1}^{12}\left(t_{1}^{8}\right)+8\left(t_{3}^{2}\right)^{2} t_{3}^{4}\left(t_{1}^{2} t_{3}^{2}\right)\right\} .
$$

It can be seen that we have simply multiplied the entries in each column including the multiplicity 2 of the type I solution and omitted all terms involving $s_{k}$. According to Theorem 3, setting $t_{k}^{2}=2$ in this expression gives the number of self-dual colorings, namely 1376 .

The cycle index formulas in Table 3 were also used in [8] with Theorems 2 and 3 to calculate the numbers of self-dual and self-complementary configurations for each individual type of solution. These results are found in Table 4. We have also included all numbers of configurations with an equal number of vertices of each color. As in the case of necklaces, these are found from Theorem 1 to be the middle coefficient of $Z(A, 1+z)$ for each choice of $A$. 
self-dual self-complementary

$m$ vertices

of each color

Octahedron

Cubactahedron

Cube

Truncated

octahedron

Truncated

cube

Truncated

cuboctohedron

Dodecahedron

Icosadodecahedron

Icosahedron
1

3

3

120

114

351120

20

280

4
2

8

6

1384

1128

6642048

140

280

10
2

30

6

57168

56846

671826149514

1648

1295266

18

TABLE 4. 2-colored polyhedra.

Rotations

identity

opposite faces

fixed (6 pairs)

opposite vertices

fixed (10 pairs)

opposite edges

fixed (15 pairs)

Reflections

The reflection that

interchanges opposite

vertices is followed by

each of the rotations

above
Number of Vertices Edges Faces

\section{Permutations}

$\begin{array}{cccc}1 & t_{1}^{20} & t_{1}^{30} & t_{1}^{12} \\ 24 & t_{5}^{4} & t_{5}^{6} & t_{1}^{2} t_{5}^{2} \\ 20 & t_{1}^{2} t_{3}^{6} & t_{3}^{10} & t_{3}^{4} \\ 15 & s_{2}^{2} t_{2}^{8} & t_{1}^{2} s_{2}^{2} t_{2}^{12} & s_{2}^{2} t_{2}^{4} \\ 1 & s_{2}^{10} & s_{2}^{15} & s_{2}^{6} \\ & & & \\ 24 & s_{10}^{2} & s_{10}^{3} & s_{2} s_{10} \\ 20 & s_{2} s_{6}^{3} & s_{6}^{5} & s_{6}^{2} \\ 15 & t_{1}^{4} t_{2}^{8} & t_{1}^{4} s_{2} t_{2}^{12} & t_{1}^{4} t_{2}^{4}\end{array}$

TABLE 5. Cycle structures of the group of the dodecahedron. 
The same theoretical approach applies to coloring the vertices of the dodecahedron and many other related polyhedra. In Table 5 we display the terms in the cycle index of three representations of the group of the dodecahedron. Once the terms for the rotations are found, the same rules (a) and (b) above are used to find the entries for the reflections. The dual of the dodecahedron is the icosahedron and so the last column in Table 5 gives its cycle index. The line graph of the dodecahedron is called the icosadodecahedron and its cycle index is given by the terms in the preceding column of the table.

The cycle indexes for many other related configurations were calculated in [8] and used to compute the numbers of self-dual colorings, self-complementary colorings and colorings with an equal number of vertices of each color. Table 4 includes the results for the three configurations of Table 5.

3. Boolean functions. Pólya [11] calculated the number of boolean functions composed of $n$ statements for $n \leq 4$ by observing that the problem was equivalent to counting the number of ways of coloring the vertices of the $n$-cube $Q_{n}$ with two different colors, say black and white. A complete treatment of this enumeration problem requires a formula for the cycle index of the group of $Q_{n}$. Slepian [15] found a formula for this cycle index, which can also be obtained by applying an algorithm of Harrison and High [7]. A compact general formula, found in the book Graphical Enumeration [5, p. 112] and verified in [9], can also be used and we shall follow the notation and definitions of the latter two references in the present discussion.

A boolean function is self-dual if it is unchanged when all of its $n$ statements and the proposition itself are negated. Therefore these correspond to 2-colorings of the vertices of the $n$-cube in which opposite vertices have different colors. To count these using Theorem 3 we require the cycle index of the group of the $n$-cube to be expressed in two sets of variables: $s_{k}$ for self-dual cycles and $t_{k}^{2}$ for pairs of dual cycles.

The group $Q_{n}$ of the $n$-cube can be viewed as the exponentiation group $\left[S_{2}\right]^{S_{n}}$. The object set of this exponentiation group is the set of functions from $\{1, \ldots, n\}$ into $\{0,1\} ; S_{n}$ acts on the domain, and $S_{2}$ acts independently on the image of each domain element. The duality involution is obtained from the identity on the domain and the transposition on each image. That is, if $f:\{1, \ldots, n\} \rightarrow\{0,1\}$ then $f^{*}(x)=1-f(x)$ for all $1 \leq x \leq n$. It is easy to see that this involution is in the center of $\left[S_{2}\right]^{S_{n}}$.

According to [9], in order to find the ordinary cycle index of $\left[S_{2}\right]^{S_{n}}$ we first substitute the operators $I_{m}$ for each variable $a_{m}$ in the cycle index 
$Z\left(S_{n}\right)$ of the symmetric group. To obtain $Z\left(\left[S_{2}\right]^{S_{n}}\right)$ we let $Z\left(S_{n} ; I_{1}, \ldots, I_{n}\right)$ act on $Z\left(S_{2}\right)$ as required in Theorem 2 of [9].

For the $n$-cube these operators are defined by

$$
I_{m}\left(\prod_{k=1}^{2} a_{k}^{j_{k}}\right)=\prod_{r=1}^{2^{m}} a_{r}^{i_{r}}
$$

where

$$
i_{r}=\frac{1}{r} \sum_{w \mid r} \mu\left(\frac{r}{w}\right)\left(\sum k j_{k}\right)^{(m, w)}
$$

and the second sum is over all divisors $k$ of $w /(m, w)$. Here $(m, w)$ denotes the greatest common divisor of $m$ and $w$. However, we wish to express the right side of (3) in terms of the two variables $s_{r}$ and $t_{r}$. That is, each $a_{r}^{i_{r}}$ must be expressed as $s_{r}^{u_{r}} t_{r}^{v_{r}}$, with $i_{r}=u_{r}+v_{r}$.

Suppose $\alpha=(12 \cdots m)$, a cycle of length $m$, and $(\alpha, \tau)$ is a typical element of $\left[S_{2}\right]^{S_{m}}$. Then, following the notation of our paper [9] on the exponentiation group, we have

$$
\beta=\tau(m) \tau(m-1) \cdots \tau(2) \tau(1),
$$

and, of course, since $\beta \in S_{2}$ we can write $\beta=(0)(1)$ or $\beta=(01)$. In order to calculate $u_{r}$ we shall provide a formula for the number of self-dual transpositions in the powers of $(\alpha, \tau)$. This will enable us to find $u_{r}$ for any $r$. We start by establishing the pattern of dual elements in a self-dual cycle under fairly general conditions.

LemMa. Let $*$ be an involution on $X$ with no fixed points and let $\alpha$ be a permutation of $X$ which commutes with $*$. Then in any self-dual cycle of length $i$ of $\alpha, i$ is even and $\alpha^{i / 2}$ maps each element to its dual.

Proof. Let us say that for some $x \in X$ we have $\alpha^{i} x=x$ and $\alpha^{k} x \neq x$ for $1 \leq k<i$. If the cycle containing $x$ is self-dual then $\alpha^{m} x=x^{*}$ for some $1 \leq m \leq i$. In fact $m<i$ since $*$ has no fixed points. Now

$$
\alpha^{2 m} x=\alpha^{m} x^{*}=\left(\alpha^{m} x\right)^{*}=x^{* *}=x,
$$

so $i$ divides $2 m$. Thus $i=2 m$.

Now it is convenient to denote the number of self-dual cycles of length $r$ in $(\alpha, \tau)^{p}$ by $u_{r}(\alpha, \tau)^{p}$. By the Lemma we have the relation

$$
u_{2}(\alpha, \tau)^{r}=\sum^{\prime} k u_{2 k}(\alpha, \tau),
$$

where the sum is over all $k \mid r$ such that $r / k$ is odd. This is because the $r$ th power of a self-dual $2 k$-cycle is the product of $k$ self-dual transpositions 
just if $r$ is an odd multiple of $k$. By Möbius inversion, (4) can be solved for $u_{2 r}(\alpha, \tau)$ explicitly:

$$
u_{2 r}(\alpha, \tau)=\frac{1}{r} \sum^{\prime} \mu\left(\frac{r}{k}\right) u_{2}(\alpha, \tau)^{k},
$$

with the same sum over $k$ as in (4).

It only remains to establish the numbers of self-dual transpositions, which are given by

(6) $u_{2}(\alpha, \tau)^{p}= \begin{cases}2^{(m, p)-1} & \text { if } \beta=(0)(1) \text { and } m /(m, p) \text { is even, or } \\ & \text { if } \beta=(01) \text { and }[m, p] /(m, p) \text { is odd } \\ 0 \quad \text { otherwise. }\end{cases}$

Here $[m, p]$ denotes the least common multiple of $m$ and $p$. Recall that $\alpha=(12 \cdots m)$ and $\beta$ is the product of the $m$ coordinate permutations from $S_{2}$. On the cartesian product $\{1,2, \ldots, m\} \times\{0,1\}$ we see that if $\beta=(0)(1)$ then $(\alpha, \tau)$ induces two cycles of the form

$$
\left(\langle 1,0\rangle\left\langle 2, \varepsilon_{2}\right\rangle \cdots\left\langle m, \varepsilon_{m}\right\rangle\right)
$$

and its dual

$$
\left(\langle 1,1\rangle\left\langle 2, \varepsilon_{2}^{*}\right\rangle \cdots\left\langle m, \varepsilon_{m}^{*}\right\rangle\right) .
$$

Now we seek to partition the cartesian product into two functional subsets $B$ and $C$ which are interchanged by $(\alpha, \tau)$. We can start by choosing $B$ or $C$ to contain the pair $\langle 1,0\rangle$. If we say that $\langle 1,0\rangle \in B$, then the partition is determined by $\langle 1,1\rangle \in C$ and the requirement that in each cycle the pairs must alternate between those in $B$ and those in $C$. This is not possible if $n$ is odd. If $n$ is even, the partition is possible in only one way since it doesn't matter which set is called $B$ and which is called $C$. If we now consider the $p$ th power of $(\alpha, \tau)$, each of the original $m$-cycles of pairs is broken into the product of $(p, m)$ disjoint cycles, each of length $m /(p, m)$. On choosing a partition into functional sets $B$ and $C$ interchanged by $(\alpha, \tau)^{p}$, each dual pair of cycles presents the same binary choice that was available when $p=1$. Thus there are $2^{(m, p)-1}$ different self-dual transpositions in $(\alpha, \tau)$ if $m /(p, m)$ is even, since the two functional sets can have their names interchanged without affecting the transposition. Of course there are none if $m /(p, m)$ is odd.

On the other hand, if $\beta=(01)$ then $(\alpha, \tau)$ induces the single cycle $\left(\langle 1,0\rangle\left\langle 2, \varepsilon_{2}\right\rangle \cdots\left\langle m, \varepsilon_{m}\right\rangle\langle 1,1\rangle\left\langle 2, \varepsilon_{2}^{*}\right\rangle \cdots\left\langle m, \varepsilon_{m}^{*}\right\rangle\right)$. A partition into two functional subsets $B, C$ which are interchanged by $(\alpha, \tau)$ would have alternate pairs of the cycle in $B$ and alternate pairs in $C$. We can specify 
that one pair be in either $B$ or $C$. Suppose $\langle 1,0\rangle \in B$. Then $\langle 1,1\rangle \in C$ and so $m$ must be odd. If $m$ is odd there is just one such division with $\langle 1,0\rangle \in B$. As interchanging the names of $B$ and $C$ makes no difference to the self-dual transposition determined, there is just one such induced by $(\alpha, \tau)$ in this case if $m$ is odd and none if $m$ is even. Now the $p$ th power of $(\alpha, \tau)$ permutes our set of pairs in $(2 m, p)$ disjoint cycles, each of length $2 m /(2 m, p)$. If the lengths are odd then typical dual pairs $\langle 1,0\rangle$ and $\langle 1,1\rangle$ fall into different cycles of $(\alpha, \tau)^{p}$. This was seen in the case $\beta=(0)(1)$ to require even length cycles in order to allow a partition into a self-dual functional subsets. Accordingly the latter is only possible if $2 m /(2 m, p)$ is even, when dual pairs like $\langle 1,0\rangle$ and $\langle 1,1\rangle$ fall into the same cycle. Then, as seen for $p=1$, the half cycle length $m /(2 m, p)$ must be odd. This implies that $m$ and $p$ are divisible by equal powers of 2 , or equivalently $[m, p] /(m, p)$ is odd. In that case the number $(2 m, p)$ of cycles can be written $(m, p)$. In choosing a partition into functional subsets $B, C$ to be duals interchanged by $(\alpha, \tau)^{p}$, each of these cycles offers a binary choice. However in the end the names of $B$ and $C$ can be interchanged, and so there are exactly $2^{(m, p)-1}$ self-dual transpositions in $(\alpha, \tau)^{p}$. That concludes the justification of (6).

We can now use equations (3), (5) and (6) to express $I_{m}\left(a_{1}^{2}\right)$ and $I_{m}\left(a_{2}\right)$, i.e., $\beta=(0)(1)$ and (01) respectively, in terms of $s_{k}$ and $t_{k}$. For small values of $m$ the results are summarized in Table 6 .

\begin{tabular}{l|l|l}
$m$ & $I_{m}\left(a_{1}^{2}\right)$ & $I_{m}\left(a_{2}\right)$ \\
\hline 1 & $t_{1}^{2}$ & $s_{2}$ \\
2 & $t_{1}^{2} s_{2}$ & $s_{4}$ \\
3 & $t_{1}^{2} t_{3}^{2}$ & $s_{2} s_{6}$ \\
4 & $t_{1}^{2} t_{4}^{2} s_{2} s_{4}$ & $s_{8}^{2}$ \\
5 & $t_{1}^{2} t_{5}^{6}$ & $s_{2} s_{10}^{3}$ \\
6 & $t_{1}^{2} t_{3}^{2} t_{6}^{8} s_{2} s_{6}$ & $s_{4} s_{12}^{5}$
\end{tabular}

TABLE 6. Values of $I_{m}$.

In [9] it was shown that the cycle index of the exponentiation group $[B]^{A}$ is found by forming cartesian products of the polynomials $I_{m}(Z(B)$ ). We need to adapt the cartesian product to distinguish self-dual cycles from others. The product $u_{i} \times v_{j}$ (where $u$ and $v$ are each either $t$ or $s$ ) represents an $i$-cycle and a $j$-cycle of functions on disjoint domains. We obtain a function on the union of these domains by choosing one function 
from each cycle. Evidently such a function is mapped back to itself only after $[i, j]$ steps. There are $i j$ pairs of functions and hence $i j /[i, j]=(i, j)$ disjoint cycles of functions on the union domain. For a cycle to be self-dual we know from the Lemma that any function in it must be mapped to its dual in exactly $[i, j] / 2$ steps. This requires each portion to be mapped to its dual, i.e., each of $u$ and $v$ must be $s$ and $[i, j] / 2$ must be an odd multiple of both $i / 2$ and $j / 2$. This is equivalent to $[i, j] /(i, j)$ being odd since $i$ and $j$ must be even when $u$ and $v$ are $s$. Thus for a pair of variables we can express the cartesian product rule as

$$
u_{i} \times v_{j}= \begin{cases}s_{[i, j]}^{(i, j)} & \text { if } u=v=s \text { and }[i, j] /(i, j) \text { is odd } \\ t_{[l, j]}^{(i, j)} & \text { otherwise. }\end{cases}
$$

In taking the cartesian product between pairs of monomials, each monomial represents functions on a certain domain being permuted in cycles of various lengths. Thus for functions on the unions of these two domains we should multiply together the contributions on all pairs of cycles, one from each monomial. This is accomplished by specifying that $X$ is distributive over - in all monomials. As usual the specificiation of the carteisan product is completed by requiring that it be bilinear with respect to the addition and scalar multiplication in the ring of formal power series in $t_{1}, s_{1}, t_{2}, s_{2}, \ldots$ over the rationals.

We shall now illustrate the whole procecdure by calculating the number of self-dual boolean functions composed of 3 statements, or what is the same, the number of ways of coloring the vertices of the cube $Q_{3}$ with two colors so that opposite vertices have different colors. The automorphism group of $Q_{3}$ is the exponentiation group $\left[S_{2}\right]^{S_{3}}$ and the cycle index of this group is calculated step by step in our article [9]. Here we shall introduce the deviations which allow us to express it with the two sets of variables $s_{k}$ and $t_{k}$. First we substitute the operator $I_{m}$ for each variable $a_{m}$ in $Z\left(S_{3}\right)$ :

$$
Z\left(S_{3} ; I_{1}, I_{2}, I_{3}\right)=\frac{1}{3 !}\left(I_{1}^{3}+3 I_{1} I_{2}+2 I_{3}\right) .
$$

These terms in (8) act on $Z\left(S_{2}\right)$ as follows:

$$
\begin{aligned}
I_{1}^{3}\left(Z\left(S_{2}\right)\right) & =I_{1}\left(Z\left(S_{2}\right)\right) \times I_{1}\left(Z\left(S_{2}\right)\right) \times I_{1}\left(Z\left(S_{2}\right)\right), \\
I_{1} I_{2}\left(Z\left(S_{2}\right)\right) & =I_{1}\left(Z\left(S_{2}\right)\right) \times I_{2}\left(Z\left(S_{2}\right)\right) .
\end{aligned}
$$

From the definitions of the operators $I_{m}$ it follows that

$$
I_{m}\left(Z\left(S_{2}\right)\right)=\frac{1}{2}\left(I_{m}\left(a_{1}^{2}\right)+I_{m}\left(a_{2}\right)\right) \text {. }
$$


The right side of (10) can be expressed for $m=1,2$ and 3 in terms of $s_{k}$ and $t_{k}$ by using Table 6 . The result is

$$
\begin{aligned}
& I_{1}\left(Z\left(S_{2}\right)\right)=\frac{1}{2}\left(t_{1}^{2}+s_{2}\right), \\
& I_{2}\left(Z\left(S_{2}\right)\right)=\frac{1}{2}\left(t_{1}^{2} s_{2}+s_{4}\right), \\
& I_{3}\left(Z\left(S_{2}\right)\right)=\frac{1}{2}\left(t_{1}^{2} t_{3}^{2}+s_{2} s_{6}\right) .
\end{aligned}
$$

From (9) and the definition of the cartesian product $\times$ for polynomials we have

$$
\begin{aligned}
& I_{1}^{3}\left(Z\left(S_{2}\right)\right)=\frac{1}{2}\left(t_{1}^{2}+s_{2}\right) \times \frac{1}{2}\left(t_{1}^{2}+s_{2}\right) \times \frac{1}{2}\left(t_{1}^{2}+s_{2}\right) \\
& =\frac{1}{8}\left(t_{1}^{2} \times t_{1}^{2} \times t_{1}^{2}+3 t_{1}^{2} \times t_{1}^{2} \times s_{2}+3 t_{1}^{2} \times s_{2} \times s_{2}+s_{2} \times s_{2} \times s_{2}\right) \\
& =\frac{1}{8}\left(t_{1}^{8}+6 t_{2}^{4}+s_{2}^{4}\right), \\
& I_{1} I_{2}\left(Z\left(S_{2}\right)\right)=\frac{1}{2}\left(t_{1}^{2}+s_{2}\right) \times \frac{1}{2}\left(t_{1}^{2} s_{2}+s_{4}\right) \\
& =\frac{1}{4}\left(t_{1}^{2} \times t_{1}^{2} s_{2}+t_{1}^{2} \times s_{4}+s_{2} \times t_{1}^{2} s_{2}+s_{2} \times s_{4}\right) \\
& =\frac{1}{4}\left(t_{1}^{4} t_{2}^{2}+t_{2}^{2} s_{2}^{2}+2 t_{4}^{2}\right) .
\end{aligned}
$$

Having determined the images of $Z\left(S_{2}\right)$ under $I_{1}^{3}, I_{1} I_{2}$ and $I_{3}$ we have by linearity its image under $Z\left(S_{3} ; I_{1}, I_{2}, I_{3}\right)$ :

$$
\begin{aligned}
& Z^{*}\left(\left[S_{2}\right]^{S_{3}} ; s_{k}, t_{k}\right) \\
& \quad=\frac{1}{3 ! 2^{3}}\left\{t_{1}^{8}+6 t_{2}^{4}+s_{2}^{4}+6 t_{1}^{4} t_{2}^{2}+12 t_{4}^{2}+6 t_{2}^{2} s_{2}^{2}+8 t_{1}^{2} t_{3}^{2}+8 s_{2} s_{6}\right\} .
\end{aligned}
$$

Note that this formula agrees pleasantly with the values in Table 3 for the cube which were derived in rather a different manner. It now follows from Theorem 3 that we can calculate the number of self-dual colorings of $Q_{3}$ by setting $t_{k}^{2}=2$ and $s_{k}=0$ for each $k$ in this expression. The result is 3 and each of these configurations is shown in Figure 3.
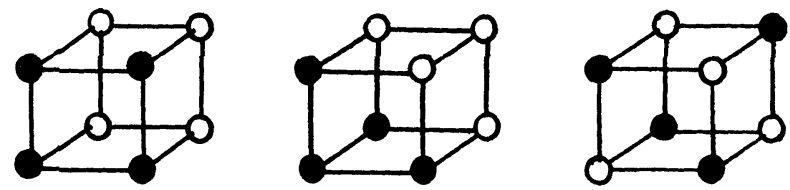

FIGURE 3. The self-dual colorings of the cube. 
The number of self-complementary colorings is obtained by setting $t_{k}=$ $s_{k}=0$ whenever $k$ is odd and $t_{k}=s_{k}=2$ when $k$ is even. Here the result is 6 and the reader can quickly find the three in addition to those already depicted in Figure 3.

We have used this method to calculate the number of self-complementary and self-dual boolean functions for $n=1$ through 6 statements and our results appear in Table 7. For comparison the number of boolean functions taking each of the two range values equally often (evenly valued) is included.

$\begin{array}{rrrr}n & \text { self-dual } & \text { self-complementary } & \text { evenly valued }{ }^{*} \\ 1 & 1 & 1 & 1 \\ 2 & 1 & 2 & 2 \\ 3 & 3 & 6 & 6 \\ 4 & 7 & 42 & 74 \\ 5 & 83 & 4094 & 169112 \\ 6 & 109950 & 98210640 & 39785643746726\end{array}$

TABLE 7. Boolean functions.

The numbers of self-complementary colorings of the $n$-cube for $n \leq 6$ also appear in [6].

\section{Related Problems}

The enumeration theorems given earlier can be modified so as to count achiral configurations when the domain group $A$ is supplied with a homomorphism onto $S_{2}$. The configurations which are simultaneously achiral and self-dual can be enumerated in this manner, and these results will appear elsewhere. However in general it appears quite difficult to enumerate those that are simultaneously achiral and self-complementary. The only case in which the latter has been accomplished is that of self-complementary achiral necklaces [10].

\section{REFERENCES}

[1] A. E. Brouwer, The enumeration of locally transitive tournaments, Report ZW 138/80, Mathematisch Centrum, Amsterdam (1980).

[2] N. G. de Bruijn, Pólya's Theory of Counting, Applied Combinatorial Mathematics (E. F. Beckenbach, ed.), Wiley, New York (1964), 144-184.

[3] Colour patterns which are invariant under a given permutation of the colours, $\mathrm{J}$. Combinatorial Theory, 2 (1967), 418-421.

\footnotetext{
* Computer programming for the reported data was performed by A. Nymeyer. The second author is grateful to the Australian Research Grants Committee for its financial support, which provided technical assistance and some of the computing equipment.
} 
[4] S. W. Golomb, Shift Register Sequences, Holden-Day, San Francisco (1967).

[5] F. Harary and E. M. Palmer, Graphical Enumeration, Academic, New York (1973).

[6] M. A. Harrison, The number of equivalence classes of boolean functions under groups containing negation, IEEE Trans. Electronic Computers EC-12 (1963), 559-561.

[7] M. A. Harrison and R. G. High, On the cycle index of a product of permutation groups, J. Combinatorial Theory, 3 (1968), 1-23.

[8] O. Knop, E. M. Palmer and R. W. Robinson, Arrangements of point charges having zero electric field gradient, Acta Cryst. Sect. A, 31 (1975), 19-31.

[9] E. M. Palmer and R. W. Robinson, Enumeration under two representations of the wreath product, Acta Math., 131 (1973), 123-143.

[10] E. M. Palmer and A. J. Schwenk, The number of self-complementary, archiral necklaces, J. Graph Theory, 1 (1977), 309-315.

[11] G. Pólya, Sur les types des propositions composees, J. Symbolic Logic, 5 (1940), 98-103.

[12] Kombinatorische Anzahlbestimmungen fur Gruppen, Graphen und chemische Verbindungen, Acta Math., 68 (1937), 145-254.

[13] R. C. Read, On the number of self-complementary graphs and digraphs, J. London Math. Soc., 38 (1963), 99-104.

[14] J. H. Redfield, The theory of group-reduced distributions, Amer. J. Math., 49 (1927), 433-435.

[15] D. Slepian, On the number of symmetry types of boolean functions of $n$ variables, Canad. J. Math., 5 (1953), 185-193.

Received September 23, 1981. Work by the first author was supported in part by a grant from the National Science Foundation. The second author was supported in part by the Australian Research Grants Committee.

Michigan State University

EAST LANSING, MI 48824

UNIVERSITY OF NEWCASTLE

N.S.W., Australia

AND

SOUTHERN ILLINOIS UNIVERSITY

CARBONDALE, IL 62901 



\section{PACIFIC JOURNAL OF MATHEMATICS \\ EDITORS}

DONALD BABBITT (Managing Editor)

University of California

Los Angeles, CA 90024

Hugo RossI

University of Utah

Salt Lake City, UT 84112

C. C. MOOre and ARThur OGus

University of California

Berkeley, CA 94720
J. Dugundi

Department of Mathematics

University of Southern California

Los Angeles, CA 90089-1113

R. FINN and H. SAMELSON

Stanford University

Stanford, CA 94305

ASSOCIATE EDITORS

$\begin{array}{lllll}\text { R. Arens } & \text { E. F. Beckenbach } & \text { B. H. Neumann } & \text { F. Wolf } & \text { K. Yoshida }\end{array}$ $(1906-1982)$

\section{SUPPORTING INSTITUTIONS}

UNIVERSITY OF ARIZONA

UNIVERSITY OF BRITISH COLUMBIA

CALIFORNIA INSTITUTE OF TECHNOLOGY

UNIVERSITY OF CALIFORNIA

MONTANA STATE UNIVERSITY

UNIVERSITY OF NEVADA, RENO

NEW MEXICO STATE UNIVERSITY

OREGON STATE UNIVERSITY
UNIVERSITY OF OREGON

UNIVERSITY OF SOUTHERN CALIFORNIA

STANFORD UNIVERSITY

UNIVERSITY OF HAWAII

UNIVERSITY OF TOKYO

UNIVERSITY OF UTAH

WASHINGTON STATE UNIVERSITY

UNIVERSITY OF WASHINGTON 


\section{Pacific Journal of Mathematics}

\section{Vol. 110, No. $1 \quad$ September, 1984}

Wojciech Abramczuk, A class of surjective convolution operators $\ldots \ldots \ldots \ldots 1$

K. Adachi, Extending bounded holomorphic functions from certain

subvarieties of a weakly pseudoconvex domain $\ldots \ldots \ldots \ldots \ldots \ldots$

Malvina Florica Baica, An algorithm in a complex field and its application

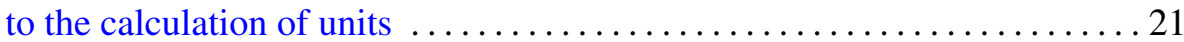

Giuliana Bianchi and Robert Cori, Colorings of hypermaps and a

conjecture of Brenner and Lyndon $\ldots \ldots \ldots \ldots \ldots \ldots \ldots \ldots \ldots \ldots \ldots \ldots$

Ronald James Evans, Determinations of Jacobsthal sums . . .......... 49

Leslie Foged, Characterizations of $\aleph$-spaces .................... 59

Nassif A. Ghoussoub and Paulette Saab, Weak compactness in spaces of

Bochner integrable functions and the Radon-Nikodým property . . . . . . 65

J. Gómez Gil, On local convexity of bounded weak topologies on Banach

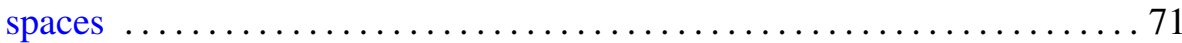

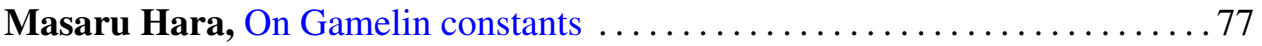

Wilfried Hauenschild, Eberhard Kaniuth and Ajay Kumar, Harmonic

analysis on central hypergroups and induced representations $\ldots \ldots \ldots 83$

Eugenio Hernandez, An interpolation theorem for analytic families of

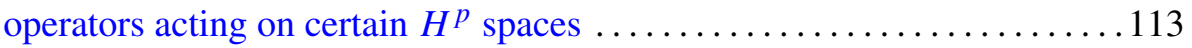

Thomas Alan Keagy, On "Tauberian theorems via block-dominated

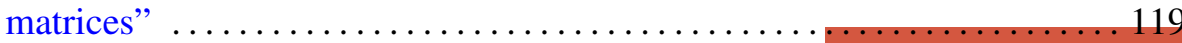

Thomas Landes, Permanence properties of normal structure $\ldots \ldots \ldots \ldots \ldots 125$

Daniel Henry Luecking, Closed ranged restriction operators on weighted

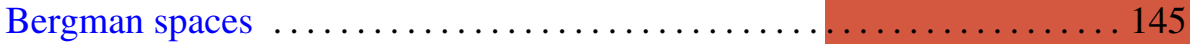

Albert Thomas Lundell, The $p$-equivalence of $\mathrm{SO}(2 n+1)$ and $\mathrm{Sp}(n) \ldots \ldots 161$

Mark D. Meyerson, Remarks on Fenn's "the table theorem" and Zaks' "the chair theorem" ..................................... 167

Marvin Victor Mielke, Homotopically trivial toposes . . . . . . . . . . 171

Gerard J. Murphy, Hyperinvariant subspaces and the topology on Lat A . . 183

Subhashis Nag, On the holomorphy of maps from a complex to a real manifold

Edgar Milan Palmer and Robert William Robinson, Enumeration of self-dual configurations ................................. 203

John J. Walsh and David Clifford Wilson, Continuous decompositions

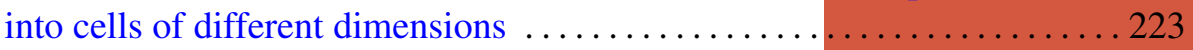

Walter John Whiteley, Infinitesimal motions of a bipartite framework .....233 